Ann. Génét. Sél. anim. I977, 9 (I),I5-I6.

\title{
Séminaire du département de génétique animale de l'I.N.R.A.
}

\author{
ESTIMATION DE LA VALEUR GÉNÉTIQUE \\ DES REPRODUCTEURS
}

(6-7 Octobre I976)

Toulouse, France

Les 6 et 7 octobre derniers s'est déroulé au centre de recherches de Toulouse le séminaire annuel du Département de Génétique animale de l'I.N.R.A. Le thème choisi était celui de l'estimation de la valeur génétique des reproducteurs. Les contributions des chercheurs avaient été réparties suivant les trois rubriques suivantes :

\section{I. - Modèles statistiques et génétiques (animateur G. Lefort : I.N.A.-Paris-Grignon, Paris)}

Après un rappel synthétique du modèle général d'estimation de la valeur génétique (breeding value) pour un cu plusieurs caractères effectué par RouviER (I.N.R.A., Toulouse), les orateurs suivants ont abordé différentes situations mettant en défaut certaines hypothèses et développements de ce modèle aujourd'hui classique et largement utilisé. DEMPFLE (Munich, R.F.A.) a fait part de sa vision bayesienne du modèle d'Henderson; Chesnars (I.N.R.A., Jouy) a montré l'intérêt pratique de cette démarche de cumul d'informations a priori et $a$ posteriori pour l'évaluation des taureaux laitiers avec l'existence actuelle de sous-populations génétiquement distinctes dans le temps et dans l'espace. Les problèmes particuliers que pose le traitement des variables discrètes ont été examinées ensuite par RAzUNGLES (I.N.R.A., Toulouse). L'abandon de l'hypothèse de panmixie, homogamie notamment à laquelle est confrontée LANGLOrs (I.N.R.A., Jouy) dans ses études sur le pur sang anglais, implique, comme l'a indiqué CHEVALET (I.N.R.A., Toulouse de reconsidérer le modèle statistique d'établissement des indices compte tenu des changements intervenant dans les rolations entre apparentés et des difficultés soulevées par le rôle particulier que joue alors le milieu. Enfin, une approche probabiliste relative aux dispositifs expérimentaux permettant de garder le meilleur a été présentée par Paule RENAUd (I.N.A.Paris-Grignon, Paris).

\section{2. - Pondération des valeurs génétiques dans l'objectif de sélection}

(animateur L. OlLIVIER : I.N.R.A.-Jouy)

Après les réflexions générales de Schlote (Hohenheim, R.F.A.) et TAYLoR (A.B.R.O., R.U.) sur le choix des caractères à prendre en compte dans le cas d'un objectif de sélection multiple, une discussion critique s'est engagée sur le mode d'évaluation des pondérations $\left(a_{i}\right)$ des caractères dans l'objectif $\left(\mathrm{H}=\Sigma a_{i} g_{i}\right)$. ELSEN (I.N.R.A., Jouy) a proposé une méthode de calcul très générale des pondérations quand les caractères s'expriment sur des animaux distincts et à des moments différents. Cette méthode a d'ailleurs été appliquée à la sélection des races bovines mixtes à la fois pour le lait et la viande. L'imprécision avec laquelle sont estimées ces pondérations peut entraîner une chute de l'efficacité réelle de la sélection sur indice. VANDEPITTE (Louvain, Belgique) en collaboration avec HAZEL a abordé ce problème à travers l'exemple du contrôle 
combiné chez le porc, pour un objectif incluant 7 caractères relatifs à la taille de portée au sevrage, aux performances d'engraissement et d'abattage. Face à la difficulté d'apprécier objectivement ces pondérations dans bien des cas, on peut alors faire appel, comme l'a suggéré MaLLARD (E.N.S.A., Rennes) aux techniques d'indexation avec contraintes, mais non sans certaines précautions.

\section{3. - Incidence de l'information prise en compte en vue de l'estimation de la valeur génétique (animateur M. Poutous : I.N.R.A., Jouy)}

L'objectif de sélection $\mathrm{H}$ étant fixé, il reste à le prédire à partir de l'information concernant divers caractères, disponible sur les reproducteurs, leurs apparentés et les populations auxquelles ils appartiennent. Aussi, une des questions fondamentales qui se posent, réside dans le choix des variables prédictrices à utiliser. On peut s'interroger notamment, comme l'a fait $B$. PouJARDIEU dans le cadre du contrôle de descendance des lapins mâles de souche chair, sur les conséquences de l'abandon de caractères onéreux à mesurer comme la consommation d'aliments et certaines caractéristiques d'abattage. Mais si l'abandon de certaines variables prédictrices peut apparaître a priori trop préjudiciable à l'efficacité de la sélection, encore faut-il, comme le prouvent les études de Hill et de Sales présentées par le Robertson (Edimbourg, R.U.), être prudent dans les conclusions compte tenu des erreurs commises dans l'estimation des paramètres génétiques et phénotypiques. C'est ainsi que par sélection individuelle pour un caractère, on peut en arriver, par l'adjonction d'un autre caractère - n'intervenant pas dans l'objectif mais retenu seulement pour l'information qu'il apporte sur le premier - à croire augmenter par là le progrès génétique alors qu'en réalité, ce faisant, on le diminuera. En plus des erreurs de précision dues à l'estimation des paramètres génétiques et phénotypiques, le progrès génétique réel va aussi fluctuer autour de son espérance du fait, d'une part, du recours à la technique de régression pour le calcul de l'indice et d'autre part, du contrôle et de la sélection de nombres limités de reproducteurs. Aussi Razungles s'est-il attaché à faire le point sur l'incidence de ces différentes sources d'erreurs dans la prévision du progrès génétique. Enfin, la concrétisation de ces problèmes méthodologiques est apparue à travers le bilan dressé par FoulLEY (I.N.R.A., Jouy) des systèmes français de qualification des reproducteurs auxquels ont contribué activement les chercheurs du département de génétique animale, aussi bien au stade de la conception qu'à celui de la réalisation.

C'est pourquoi, vu l'intérêt soulevé par le thème de ce séminaire, aussi bien chez les chercheurs de génétique animale que chez nos collègues d'amélioration des plantes et de la biométrie les Annales de Génétique et de sélection animale présentent in extenso les rapports les plus originaux de ce séminaire qui n'ont pas fait l'objet de publication jusqu'à ce jour.

\section{J. L. FOUliey, C. CHEVAlet.}

